FACTA UNIVERSITATIS (NIŠ)

Ser. Math. Inform. Vol. 36, No 2 (2021), 365-375

https://doi.org/10.22190/FUMI200803027B

Original Scientific Paper

\title{
ON A CERTAIN TRANSFORMATION IN ALMOST CONTACT METRIC MANIFOLDS
}

\author{
Gherici Beldjilali ${ }^{1}$ and Mehmet Akif $\mathrm{Akyol}^{2}$ \\ 1 Laboratory of Quantum Physics and Mathematical Modeling (LPQ3M), \\ University of Mascara, Mascara, Algeria \\ 2 Faculty of Science and Arts, Department of Mathematics, \\ 12000 Bingöl, Turkey
}

\begin{abstract}
In this work, we have investigated a new deformation of almost contact metric manifolds. New relations between classes of 3-dimensional almost contact metric have been discovered. Several concrete examples are discussed.

Key words: Sasakian manifolds; Kenmotsu manifolds; cosymplectic manifolds.
\end{abstract}

\section{Introduction}

The lifts of geometrical objects, functions, vector fields, 1-forms etc., on any manifold have important role in differential geometry. For example, they are used to define the different geometric structures. Manifolds equipped with certain differentialgeometric structures possess rich geometric structures and such manifolds have been studied widely in differential geometry. Indeed, almost contact manifolds and relations between such manifolds have been studied extensively by many authors.

The construction of almost contact metric structures (Sasakian, Kenmotsu, cosymplectic, etc.) from other almost contact metric structures on a given $(2 n+1)$ dimensional manifold $M$, in general, a non-trivial problem. The more interesting and well-known results correspond with the 3 -dimensional case.

Received August 3, 2020, accepted May 26, 2021

Communicated by Cornelia-Livia Bejan

Corresponding Author: Mehmet Akif Akyol, Faculty of Science and Arts, Department of Mathematics, 12000 Bingöl, Turkey | E-mail: mehmetakifakyol@bingol.edu.tr

2010 Mathematics Subject Classification. Primary 53C15, 53C25; Secondary 53C55, 53D25

(C) 2021 by University of Niš, Serbia | Creative Commons License: CC BY-NC-ND 
The notion of a D-homothetic deformation on a contact metric manifold was introduced by Tanno [12]. Next, A. Sharfuddin and S. I. Hussain [11] gave a study on conformal transformation of almost contact structures.

In 1992, J. C. Marrero [7] proved that with certain deformation, we can get a trans-Sasakian structure starting from a Sasakian one. In [1], generalized Dconformal deformations are applied to trans-Sasakian manifolds where the covariant derivatives of the deformed metric is evaluated under the condition that the functions used in deformation depend only on the direction of the characteristic vector field of the trans-Sasakian structure. Other similar deformations are studied in $[2,3,6]$.

Recently, Özdemir et al. [9], investigated the generalized D-conformal deformations of nearly K-cosymplectic, quasi-Sasakian and $\beta$-Kenmotsu manifolds. They analyzed how the class of almost contact metric structures changes. There exists several type of deformations of almost contact metric structures. These different known deformations are mainly based on a deformation of the Riemannian metric.

The present paper deals with the deformation of the structural tensor $\varphi$ and metric tensor $g$ at the same time, which allows us to define new relations between almost contact metric structures. The paper is organized in the following way.

Section 2 is devoted to the background of the structures which will be used in the subsequent sections to make the paper self-contained. In Section 3, we have introduced a new deformation of almost contact metric structures using a function and a 1-form and prove some basic properties. In Section 4, we focused on the case of three-dimensional geometric structures and have shown how to construct some basic structures with concrete examples. In the last Section, we constructed the examples of almost contact manifolds starting from another class of examples of almost contact manifolds, based on the three types (Sasakian, Kenmotsu, cosymplectic).

\section{Preliminaries}

An odd-dimensional Riemannian manifold $\left(M^{2 n+1}, g\right)$ is said to be an almost contact metric manifold if there exists on $M$, a $(1,1)$ tensor field $\varphi$, a vector field $\xi$ (called the structure vector field) and a 1-form $\eta$ such that

(2.1) $\eta(\xi)=1, \varphi^{2}(X)=-X+\eta(X) \xi \quad$ and $\quad g(\varphi X, \varphi Y)=g(X, Y)-\eta(X) \eta(Y)$,

for any vector fields $X, Y$ on $M$. In particular, in an almost contact metric manifold we also have $\varphi \xi=0$ and $\eta \circ \varphi=0$ [13].

Such manifold is said to be a contact metric manifold if $d \eta=\phi$, where $\phi(X, Y)=$ $g(X, \varphi Y)$ is called the fundamental 2-form of $M$.

On the other hand, the almost contact metric structure of $M$ is said to be normal if

$$
N^{(1)}(X, Y)=[\varphi, \varphi](X, Y)+2 \mathrm{~d} \eta(X, Y) \xi=0,
$$

for any $X, Y$ on $M$, where $[\varphi, \varphi]$ denotes the Nijenhuis torsion of $\varphi$, given by

$$
[\varphi, \varphi](X, Y)=\varphi^{2}[X, Y]+[\varphi X, \varphi Y]-\varphi[\varphi X, Y]-\varphi[X, \varphi Y] .
$$


In [10], the author proves that $(\varphi, \xi, \eta, g)$ is trans-Sasakian structure if and only if it is normal and

$$
\mathrm{d} \eta=\alpha \phi, \quad \mathrm{d} \phi=2 \beta \eta \wedge \phi,
$$

where d denotes the exterior derivative, $\alpha=\frac{1}{2 n} \delta \phi(\xi), \beta=\frac{1}{2 n} \operatorname{div} \xi$ and $\delta$ is the codifferential of $g$.

It is well known that the trans-Sasakian condition may be expressed as an almost contact metric structure satisfying

$$
\left(\nabla_{X} \varphi\right) Y=\alpha(g(X, Y) \xi-\eta(Y) X)+\beta(g(\varphi X, Y) \xi-\eta(Y) \varphi X) .
$$

From this formula, one can easily obtain

$$
\begin{gathered}
\nabla_{X} \xi=-\alpha \varphi X-\beta \varphi^{2} X \\
\left(\nabla_{X} \eta\right) Y=\alpha g(X, \varphi Y)+\beta g(\varphi X, \varphi Y) .
\end{gathered}
$$

It is clear that a trans-Sasakian manifold of type $(1,0)$ is a Sasakian manifold and a trans-Sasakian manifold of type $(0,1)$ is a Kenmotsu manifold. A trans-Sasakian manifold of type $(0,0)$ is called a cosymplectic manifold. More generally, a transSasakian structure $(\varphi, \xi, \eta, g)$ on $M$ is said to be

$$
\left\{\begin{array}{l}
(a): \alpha-\text { Sasaki if } \beta=0, \\
(b): \beta-\text { Kenmotsu if } \alpha=0, \\
(c): \text { Cosymplectic if } \alpha=\beta=0,
\end{array}\right.
$$

where $\alpha$ and $\beta$ are two functions.

The relations between trans-Sasakian, $\alpha$-Sasakian and $\beta$-Kenmotsu structures were discussed by Marrero [7].

Proposition 2.1. [7] A trans-Sasakian manifold of dimension $\geq 5$ is either $\alpha$ Sasakian, $\beta$-Kenmotsu or cosymplectic.

For more background on almost contact metric manifolds, we recommend the reference [4] and [5].

\section{Deformation of almost contact metric structures}

Let $(\varphi, \xi, \eta, g)$ be an almost contact metric structure on $M^{2 n+1}$. For any $X, Y$ on $M$, we mean a change of structure tensors of the form

(3.1) $\tilde{\varphi} X=\varphi X+\theta(\varphi X) \xi, \quad \tilde{\xi}=\xi, \quad \tilde{\eta}=\eta-\theta, \quad \tilde{g}(\tilde{\varphi} X, \tilde{\varphi} Y)=f g(\varphi X, \varphi Y)$,

where $\theta$ is a 1-form orthogonal to $\eta$ and $f$ a positive function on $M$.

Proposition 3.1. The structure $(\tilde{\varphi}, \tilde{\xi}, \tilde{\eta}, \tilde{g})$ is an almost contact metric structure.

Proof. The proof follows by a usual calculation, by using (2.1). 
In particular, if $\theta=0$ then we get

$$
\tilde{g}=f g+(1-f) \eta \otimes \eta
$$

and this deformation was studied by Marrero [7].

Remark 3.1. In this new deformation we required the orthogonality between $\theta$ and $\eta$. But, if we take $\theta=(1-h) \eta$ with $h$ a function on $M$, we get

$$
g=f g+\left(h^{2}-f\right) \eta \otimes \eta .
$$

This deformation appeared in [1]. In addition, if $f=1$ then we have D-isometric deformation [3], but for $h=f$ we get the deformation of Blair [6] and for $h=f=a$ where $a$ is a positive constant, we obtain D-homothetic deformation [12].

We denote the tensor field of type $(1,2)$ by $\tilde{N}^{(1)}$ on $M$ defined for any $X, Y$ on $M$ by

$$
\tilde{N}^{(1)}(X, Y)=[\tilde{\varphi}, \tilde{\varphi}](X, Y)+2 \mathrm{~d} \tilde{\eta}(X, Y) \xi,
$$

where

$$
[\tilde{\varphi}, \tilde{\varphi}](X, Y)=\tilde{\varphi}^{2}[X, Y]+[\tilde{\varphi} X, \tilde{\varphi} Y]-\tilde{\varphi}[\tilde{\varphi} X, Y]-\tilde{\varphi}[X, \tilde{\varphi} Y] .
$$

By long direct calculation, using (3.1) one can get

$$
\begin{aligned}
\tilde{N}^{(1)}(X, Y) & =N^{(1)}(X, Y)+\theta\left(N^{(1)}(X, Y)\right) \xi \\
& -\theta(\varphi X)\left(N^{(3)}(Y)+\theta\left(N^{(3)}(Y)\right) \xi\right)-\theta(\varphi Y)\left(N^{(3)}(X)+\theta\left(N^{(3)}(X)\right) \xi\right) \\
(3.2) & +2 \mathrm{~d} \theta(\tilde{\varphi} X, \tilde{\varphi} Y) \xi-2 \mathrm{~d} \theta(X, Y) \xi
\end{aligned}
$$

with $N^{(3)}$ is a tensor field on $M$ given by

$$
N^{(3)}(X)=\left(L_{\xi} \varphi\right)(X)=\varphi[X, \xi]-[\varphi X, \xi],
$$

where $L_{\xi}$ denotes the Lie derivative with respect to the vector field $\xi$.

Proposition 3.2. Let $(\varphi, \xi, \eta, g)$ be a normal almost contact metric structure on $M$. The almost contact metric structure $(\tilde{\varphi}, \tilde{\xi}, \tilde{\eta}, \tilde{g})$ is normal if and only if

$$
\mathrm{d} \theta(\varphi X, \varphi Y)=\mathrm{d} \theta(X, Y)
$$

for any $X, Y \in \Gamma(T M)$.

Proof. Firstly, we have

$$
N^{(1)}(X, Y)=0 \Rightarrow N^{(1)}(\varphi X, \xi)=[\xi, \varphi X]-\varphi[\xi, X]=N^{(3)}(X)=0 .
$$

So, if $(\varphi, \xi, \eta, g)$ is normal then from (3.2), we obtain

$$
\tilde{N}^{(1)}(X, Y)=2 \mathrm{~d} \theta(\tilde{\varphi} X, \tilde{\varphi} Y) \xi-2 \mathrm{~d} \theta(X, Y) \xi .
$$


Suppose that

$$
\mathrm{d} \theta(\varphi X, \varphi Y)=\mathrm{d} \theta(X, Y) .
$$

For $Y=\xi$ we get for any $X$ on $M$,

$$
\mathrm{d} \theta(X, \xi)=0 .
$$

Applying (3.4) and (2.1) in (3.3) we obtain $\tilde{N}^{(1)}(X, Y)=0$.

For the inverse, suppose that $\tilde{N}^{(1)}=0$ and taking $Y=\xi$ we obtain for any $X$ on $M$,

$$
\mathrm{d} \theta(X, \xi)=0 .
$$

Applying (3.5) in (3.3) we get

$$
\mathrm{d} \theta(\varphi X, \varphi Y)=\mathrm{d} \theta(X, Y) .
$$

Corollary 3.1. Let $(\varphi, \xi, \eta, g)$ be a normal almost contact metric structure on $M$. $(\tilde{\varphi}, \tilde{\xi}, \tilde{\eta}, \tilde{g})$ is normal if one of the following four conditions is satisfied

$$
\theta=0, \quad \theta=\mathrm{d} h, \quad \mathrm{~d} \theta=0, \quad \mathrm{~d} \theta=\sigma \phi,
$$

where $h, \sigma$ are two functions on $M$.

Using the Koszul formula for the Levi-Civita connection of a Riemannian metric, one can obtain the following:

Proposition 3.3. Let $\nabla$ and $\tilde{\nabla}$ denote the Levi-Civita connections of $g$ and $\tilde{g}$ respectively. For any $X$ and $Y$ on $M$, we have the relation:

$$
\begin{aligned}
\tilde{g}\left(\tilde{\nabla}_{X} Y, Z\right) & =\tilde{g}\left(\nabla_{X} Y, Z\right)+\frac{1}{2}(X(f) g(Y, Z)+Y(f) g(X, Z)-Z(f) g(X, Y)) \\
& -f\left(\frac{1}{2}\left(\left(\nabla_{X} \eta\right) Y+\left(\nabla_{Y} \eta\right) X\right) \eta(Z)+d \eta(X, Z) \eta(Y)+d \eta(Y, Z) \eta(X)\right) \\
& +\frac{1}{2}\left(\left(\nabla_{X} \tilde{\eta}\right) Y+\left(\nabla_{Y} \tilde{\eta}\right) X\right) \tilde{\eta}(Z)+d \tilde{\eta}(X, Z) \tilde{\eta}(Y)+d \tilde{\eta}(Y, Z) \tilde{\eta}(X) .
\end{aligned}
$$

\section{Application to three dimensional geometric structures}

In the remaining part of the paper, we focus on the case of 3-dimensional normal almost contact metric manifold. Let us mention here an important result of Olszak [8], which states that any normal almost contact metric structure is trans-Sasakian structure of type $(\alpha, \beta)$, where $2 \alpha=\operatorname{tr}(\varphi \nabla \xi)$ and $2 \beta=\operatorname{div} \xi$.

This is what leads us to consider $(\varphi, \xi, \eta, g)$ a trans-Sasakian structure of type $(\alpha, \beta)$ i.e., we have

$$
\mathrm{d} \eta=\alpha \phi, \quad \mathrm{d} \phi=2 \beta \eta \wedge \phi .
$$


In this section, we shall apply the new deformation on trans-Sasakian manifold. Since the expression of connection $\tilde{\nabla}$ is not easy, we prefer to use in our study the first and second fundamental forms.

Firstly, the fundamental 2 -form $\tilde{\phi}$ of $(\tilde{\varphi}, \tilde{\xi}, \tilde{\eta}, \tilde{g})$ is

$$
\tilde{\phi}(X, Y)=\tilde{g}(X, \tilde{\varphi} Y),
$$

One can easily obtain

$$
\tilde{\phi}=f \phi
$$

and hence

$$
\left\{\begin{array} { l } 
{ \tilde { \eta } = \eta - \theta } \\
{ \tilde { \phi } = f \phi }
\end{array} \quad \Rightarrow \quad \left\{\begin{array}{l}
\mathrm{d} \tilde{\eta}=\mathrm{d} \eta-\mathrm{d} \theta \\
\mathrm{d} \tilde{\phi}=(\mathrm{d}(\ln f)+2 \beta \eta) \wedge \tilde{\phi}
\end{array}\right.\right.
$$

Lemma 4.1. For any 3-dimensional almost contact metric manifold $(M, \tilde{\varphi}, \tilde{\xi}, \tilde{\eta}, \tilde{g})$, we have

$$
\mathrm{d}(\ln f) \wedge \tilde{\phi}=\xi(\ln f) \tilde{\eta} \wedge \tilde{\phi} .
$$

Proof. Let $\left\{\tilde{e}_{0}=\xi, \tilde{e}_{1}, \tilde{e}_{2}\right\}$ be the frame of vector fields and $\left\{\tilde{\theta}^{0}=\tilde{\eta}, \tilde{\theta}^{1}, \tilde{\theta}^{2}\right\}$ be the dual frame of differential 1-forms on $M$. Then,

$$
\tilde{\phi}=2 \tilde{e}_{2} \wedge \tilde{e}_{1},
$$

and

$$
\mathrm{d}(\ln f)=\xi(\ln f) \tilde{\eta}+\tilde{\theta}^{1}(\ln f) \tilde{e}_{1}+\tilde{\theta}^{2}(\ln f) \tilde{e}_{2} .
$$

Thus

$$
\mathrm{d}(\ln f) \wedge \tilde{\phi}=\xi(\ln f) \tilde{\eta} \wedge \tilde{\phi} .
$$

From (4.2) and Lemma 4.1, we get

$$
\text { (4.4) }\left\{\begin{array} { l } 
{ \mathrm { d } \eta = \alpha \phi } \\
{ \mathrm { d } \phi = 2 \beta \eta \wedge \phi }
\end{array} \Leftrightarrow \left\{\begin{array}{l}
\mathrm{d} \tilde{\eta}=\frac{\alpha}{f} \tilde{\phi}-\mathrm{d} \theta \\
\mathrm{d} \tilde{\phi}=2\left(\beta+\frac{1}{2} \xi(\ln f)\right) \tilde{\eta} \wedge \tilde{\phi}+2 \beta \theta \wedge \tilde{\phi} .
\end{array}\right.\right.
$$

We will discuss the different new structures according to the four cases indicated in the Corollary 3.1.

First case: For $\theta=0,(4.4)$ lead to us the following result:

Proposition 4.1. $(\varphi, \xi, \eta, g)$ is a trans-Sasakian of type $(\alpha, \beta)$ if and only if $(\tilde{\varphi}, \tilde{\xi}, \tilde{\eta}, \tilde{g})$ is a trans-Sasakian of type $\left(\frac{\alpha}{f}, \beta+\frac{1}{2} \xi(\ln f)\right)$. 
Remark 4.1. for $(\alpha, \beta)=(1,0)$, we can see immediately that the Proposition 4.2 of Marrero [7] is a particular case.

Remark 4.2. In this case, we can not get a Sasakian structure starting from a Kenmosu structure or vice versa.

Second case: For $\theta=\mathrm{d} h,(4.4)$ becomes:

$$
\text { (4.5) }\left\{\begin{array} { l } 
{ \mathrm { d } \eta = \alpha \phi } \\
{ \mathrm { d } \phi = 2 \beta \eta \wedge \phi }
\end{array} \Leftrightarrow \left\{\begin{array}{l}
\mathrm{d} \tilde{\eta}=\frac{\alpha}{f} \tilde{\phi} \\
\mathrm{d} \tilde{\phi}=2\left(\beta(1+\xi(h))+\frac{1}{2} \xi(\ln f)\right) \tilde{\eta} \wedge \tilde{\phi} .
\end{array}\right.\right.
$$

Proposition 4.2. $(\varphi, \xi, \eta, g)$ is a trans-Sasakian of type $(\alpha, \beta)$ if and only if $(\tilde{\varphi}, \tilde{\xi}, \tilde{\eta}, \tilde{g})$ is a trans-Sasakian of type $\left(\frac{\alpha}{f}, \beta(1+\xi(h))+\frac{1}{2} \xi(\ln f)\right)$.

Third case: For $\mathrm{d} \theta=0,(4.4)$ becomes:

$$
\text { (4.6) }\left\{\begin{array} { l } 
{ \mathrm { d } \eta = \alpha \phi } \\
{ \mathrm { d } \phi = 2 \beta \eta \wedge \phi }
\end{array} \quad \Leftrightarrow \quad \left\{\begin{array}{l}
\mathrm{d} \tilde{\eta}=\frac{\alpha}{f} \tilde{\phi} \\
\mathrm{d} \tilde{\phi}=2\left(\beta+\frac{1}{2} \xi(\ln f)\right) \tilde{\eta} \wedge \tilde{\phi}+2 \beta \theta \wedge \tilde{\phi} .
\end{array}\right.\right.
$$

Proposition 4.3. $(\varphi, \xi, \eta, g)$ is an $\alpha$-Sasakian if and only if $(\tilde{\varphi}, \tilde{\xi}, \tilde{\eta}, \tilde{g})$ is a transSasakian of type $\left(\frac{\alpha}{f}, \frac{1}{2} \xi(\ln f)\right)$.

Fourth case: For $\mathrm{d} \theta=\sigma \phi,(4.4)$ becomes:

$$
\left(4 . 7 \left\{\begin{array} { l } 
{ \mathrm { d } \eta = \alpha \phi } \\
{ \mathrm { d } \phi = 2 \beta \eta \wedge \phi }
\end{array} \Leftrightarrow \quad \left\{\begin{array}{l}
\mathrm{d} \tilde{\eta}=\frac{1}{f}(\alpha-\sigma) \tilde{\phi} \\
\mathrm{d} \tilde{\phi}=2\left(\beta+\frac{1}{2} \xi(\ln f)\right) \tilde{\eta} \wedge \tilde{\phi}+\frac{2}{\sigma} \beta f \theta \wedge \mathrm{d} \theta .
\end{array}\right.\right.\right.
$$

\section{Proposition 4.4.}

1) For $\theta \wedge \mathrm{d} \theta=0,(\varphi, \xi, \eta, g)$ is a trans-Sasakian of type of type $(\alpha, \beta)$ if and only if $(\tilde{\varphi}, \tilde{\xi}, \tilde{\eta}, \tilde{g})$ is a trans-Sasakian of type $\left(\frac{1}{f}(\alpha-\sigma), \beta+\frac{1}{2} \xi(\ln f)\right)$.

2) $(\varphi, \xi, \eta, g)$ is an $\alpha$-Sasakian if and only if $(\tilde{\varphi}, \tilde{\xi}, \tilde{\eta}, \tilde{g})$ is a trans-Sasakian of type $\left(\frac{1}{f}(\alpha-\sigma), \frac{1}{2} \xi(\ln f)\right)$.

Remark 4.3. Unlike the previous cases, this case is very interesting because we can get Sasakian structure starting from a Kenmotsu structure and vice versa (see Examples 5.1 and 5.2). 


\section{A class of examples}

For this construction, we rely on our Example in [2]. We denote the Cartesian coordinates in a 3 -dimensional Euclidean space $\mathbb{R}^{3}$ by $(x, y, z)$ and define a symmetric tensor field $g$ by

$$
g=\left(\begin{array}{ccc}
\rho^{2}+\tau^{2} & 0 & -\tau \\
0 & \rho^{2} & 0 \\
-\tau & 0 & 1
\end{array}\right)
$$

where $\rho$ and $\tau$ are functions on $\mathbb{R}^{3}$ such that $\rho \neq 0$ everywhere. Further, we define an almost contact metric $(\varphi, \xi, \eta)$ on $\mathbb{R}^{3}$ by

$$
\varphi=\left(\begin{array}{ccc}
0 & -1 & 0 \\
1 & 0 & 0 \\
0 & -\tau & 0
\end{array}\right), \quad \xi=\left(\begin{array}{l}
0 \\
0 \\
1
\end{array}\right), \quad \eta=(-\tau, 0,1)
$$

The fundamental 1-form $\eta$ and the 2-form $\phi$ can be expressed as,

$$
\eta=d z-\tau d x \quad \text { and } \quad \phi=-2 \rho^{2} d x \wedge d y
$$

and hence

$$
\begin{aligned}
\mathrm{d} \eta & =\tau_{2} d x \wedge d y+\tau_{3} d x \wedge d z \\
\mathrm{~d} \phi & =-4 \rho_{3} \rho d x \wedge d y \wedge d z
\end{aligned}
$$

where $\rho_{i}=\frac{\partial \rho}{\partial x_{i}}$ and $\tau_{i}=\frac{\partial \tau}{\partial x_{i}}$.

We know that the components of the Nijenhuis tensor $N_{\varphi}$ in (2.2) can be written as,

$$
N_{k j}^{i}=\varphi_{k}^{l}\left(\partial_{l} \varphi_{j}^{i}-\partial_{j} \varphi_{l}^{i}\right)-\varphi_{j}^{l}\left(\partial_{l} \varphi_{k}^{i}-\partial_{k} \varphi_{l}^{i}\right)+\eta_{k}\left(\partial_{j} \xi^{i}\right)-\eta_{j}\left(\partial_{k} \xi^{i}\right)
$$

where the indices $i, j, k$ and $l$ run over the range $1,2,3$, then by a direct computation we can verify that

$$
N_{k j}^{i}=0, \quad \forall i, j, k .
$$

implying that the structure $(\varphi, \xi, \eta, g)$ is normal. From $(2.7)$, the structure $(\varphi, \xi, \eta, g)$ is a:

(1) Sasaki when $\tau_{2}=-2 \rho^{2}$ and $\rho_{3}=\tau_{3}=0$,

(2) Cosymplectic when $\rho_{3}=0$ and $\tau_{2}=\tau_{3}=0$,

(3) Kenmotsu when $\rho_{3}=\rho$ and $\tau_{2}=\tau_{3}=0$. 
Since $\theta$ is a 1-form orthogonal to $\eta$, i.e. $\theta(\xi)=0$ then $\theta$ has the following form

$$
\theta=a d x+b d y
$$

where $a$ and $b$ are two functions on $\mathbb{R}^{3}$. Under these data and use (2.1), one can get

$$
\tilde{g}=\left(\begin{array}{ccc}
f \rho^{2}+(a+\tau)^{2} & b(a+\tau) & -(a+\tau) \\
b(a+\tau) & f \rho^{2}+b^{2} & -b \\
-(a+\tau) & -b & 1
\end{array}\right)
$$

and

$$
\tilde{\varphi}=\left(\begin{array}{ccc}
0 & -1 & 0 \\
1 & 0 & 0 \\
b & -(a+\tau) & 0
\end{array}\right), \quad \tilde{\xi}=\xi=\left(\begin{array}{l}
0 \\
0 \\
1
\end{array}\right), \quad \tilde{\eta}=(-(a+\tau),-b, 1) .
$$

Using the above cases, we get the following:

(1): Let $(\varphi, \xi, \eta, g)$ be a Sasakian structure
(a): If $\mathrm{d} \theta=0$ and $f=1$, then $(\tilde{\varphi}, \tilde{\xi}, \tilde{\eta}, \tilde{g})$ is a Sasakian structure.
(b): If $\mathrm{d} \theta=\phi$ and $f_{3}=2 f$, then $(\tilde{\varphi}, \tilde{\xi}, \tilde{\eta}, \tilde{g})$ is a Kenmotsu structure.
(c): If $\mathrm{d} \theta=\phi$ and $f_{3}=0$, then $(\tilde{\varphi}, \tilde{\xi}, \tilde{\eta}, \tilde{g})$ is a cosymplectic structure.

(2): Let $(\varphi, \xi, \eta, g)$ be a Kenmotsu structure
(a): If $\mathrm{d} \theta=-f \phi$ and $f_{3}=-2 f$, then $(\tilde{\varphi}, \tilde{\xi}, \tilde{\eta}, \tilde{g})$ is a Sasakian structure.
(b): If $\mathrm{d} \theta=0$ and $f_{3}=0$, then $(\tilde{\varphi}, \tilde{\xi}, \tilde{\eta}, \tilde{g})$ is a Kenmotsu structure.
(c): If $\mathrm{d} \theta=\phi$ and $f_{3}=-2 f$, then $(\tilde{\varphi}, \tilde{\xi}, \tilde{\eta}, \tilde{g})$ is a cosymplectic structure.

(3): Let $(\varphi, \xi, \eta, g)$ be a cosymplectic structure
(a): If $\mathrm{d} \theta=-\phi$ and $f=1$, then $(\tilde{\varphi}, \tilde{\xi}, \tilde{\eta}, \tilde{g})$ is a Sasakian structure.
(b): If $\mathrm{d} \theta=0$ and $f_{3}=2 f$, then $(\tilde{\varphi}, \tilde{\xi}, \tilde{\eta}, \tilde{g})$ is a Kenmotsu structure.
(c): If $\mathrm{d} \theta=\phi$ and $f_{3}=0$, then $(\tilde{\varphi}, \tilde{\xi}, \tilde{\eta}, \tilde{g})$ is a cosymplectic structure.

By using the above cases, we can discuss other classes of well-known almost contact metric structures.

Given the importance of Remark 4.3 and from the above examples, we will extract non-trivial examples in the following:

Example 5.1. (From Kenmotsu to Sasaki)

Taking $\rho=\mathrm{e}^{z}$ and $\tau=x$, we get

$$
g=\left(\begin{array}{ccc}
x^{2}+\mathrm{e}^{2 z} & 0 & -x \\
0 & \mathrm{e}^{2 z} & 0 \\
-x & 0 & 1
\end{array}\right), \quad \varphi=\left(\begin{array}{ccc}
0 & -1 & 0 \\
1 & 0 & 0 \\
0 & -x & 0
\end{array}\right)
$$




$$
\xi=\left(\begin{array}{l}
0 \\
0 \\
1
\end{array}\right), \quad \eta=(-x, 0,1) .
$$

It can be checked that $(\varphi, \xi, \eta, g)$ is a Kenmotsu structure.

Taking $\sigma=-f=-\mathrm{e}^{-2 z}$, we obtain $\mathrm{d} \theta=2 d x \wedge d y$, which implies

$$
\theta=a d x+b d y, \quad \text { with } \quad b_{1}-a_{2}=2, \quad \text { and } \quad a_{3}=b_{3}=0,
$$

where $a_{i}=\frac{\partial a}{\partial x_{i}}$ and $b_{i}=\frac{\partial b}{\partial x_{i}}$.

Notice that there is an infinite number of solutions for $\theta$. We will continue with the following particular solution $\theta=2 x d y$. So, we get

$$
\begin{gathered}
\tilde{g}=\left(\begin{array}{ccc}
1+x^{2} & 2 x^{2} & -x \\
2 x^{2} & 1+4 x^{2} & -2 x \\
-x & -2 x & 1
\end{array}\right), \quad \tilde{\varphi}=\left(\begin{array}{ccc}
0 & -1 & 0 \\
1 & 0 & 0 \\
2 x & -x & 0
\end{array}\right), \\
\tilde{\xi}=\xi=\left(\begin{array}{l}
0 \\
0 \\
1
\end{array}\right), \quad \tilde{\eta}=\eta=(-x,-2 x, 1) .
\end{gathered}
$$

Finally, we can verify that $(\tilde{\varphi}, \tilde{\xi}, \tilde{\eta}, \tilde{g})$ is a Sasakian structure.

Example 5.2. (From Sasaki to Kenmotsu)

Now, taking $\rho=\mathrm{e}^{x}$ and $\tau=-y \mathrm{e}^{2 x}$, we get

$$
\begin{gathered}
g=\mathrm{e}^{2 x}\left(\begin{array}{ccc}
1+4 y^{2} \mathrm{e}^{2 x} & 0 & 2 y \\
0 & 1 & 0 \\
2 y & 0 & \mathrm{e}^{-2 x}
\end{array}\right), \quad \varphi=\left(\begin{array}{ccc}
0 & -1 & 0 \\
1 & 0 & 0 \\
0 & 2 y \mathrm{e}^{2 x} & 0
\end{array}\right), \\
\xi=\left(\begin{array}{l}
0 \\
0 \\
1
\end{array}\right), \quad \eta=\left(2 y \mathrm{e}^{2 x}, 0,1\right) .
\end{gathered}
$$

It can be checked that $(\varphi, \xi, \eta, g)$ is a Sasakian structure.

Taking $\sigma=1$ and $f=\mathrm{e}^{2 z}$, we obtain $\mathrm{d} \theta=-2 \mathrm{e}^{2 x} d x \wedge d y$. So, we have numerious choices for $\theta$. Let's take $\theta=2 y \mathrm{e}^{2 x} d x$, we get

$$
\tilde{g}=\left(\begin{array}{ccc}
\mathrm{e}^{2(x+z)} & 0 & 0 \\
0 & \mathrm{e}^{2(x+z)} & 0 \\
0 & 0 & 1
\end{array}\right), \quad \tilde{\varphi}=\left(\begin{array}{ccc}
0 & -1 & 0 \\
1 & 0 & 0 \\
0 & 0 & 0
\end{array}\right), \quad \tilde{\xi}=\xi=\left(\begin{array}{l}
0 \\
0 \\
1
\end{array}\right), \quad \tilde{\eta}=d z .
$$

Finally, we can verify that $(\tilde{\varphi}, \tilde{\xi}, \tilde{\eta}, \tilde{g})$ is a Kenmotsu structure.

\section{Acknowledgement}

The author is grateful to the referee for his/her valuable comments and suggestions. 


\section{REF E R E N C E S}

1. P. Alegre and A. Carriazo: Generalized Sasakian Space Forms and Conformal Changes of the Metric. Results Math. 59 (2011), 485-493.

2. G. Beldjilali and M. Belkhelfa: Kählerian structures on $\mathcal{D}$-homothetic bi-warping. J. Geom. Symmetry Phys. 42 (2016), 1-13.

3. G. Beldjilali: Structures and D-isometric warping. HSIG, 2(1), (2020), 21-29.

4. C. P. Boyer, K. Galicki and P. Matzeu: On Eta-Einstein Sasakian Geometry. Comm.Math. Phys. 262 (2006), 177-208.

5. D. E. Blair: Riemannian Geometry of Contact and Symplectic Manifolds. Progress in Mathematics. Vol. 203, Birhauser, Boston, 2002.

6. D. E. BlAIR: D-homothetic warping and applications to geometric structures and cosmology. African Diaspora Journal of Math. 14 (2013), 134-144.

7. J. C. Marrero: The local structure of trans-Sasakian manifolds. Annali di Matematica Pura ed Applicata. 162 (1992), 77-86.

8. Z. OlsZaK: Normal almost contact manifolds of dimension three. Annales Pol. Math. XLVII (1986), 41-50.

9. N. Özdemir, S. Aktay and M. Solgun: On Generalized D-Conformal Deformations of Certain Almost Contact Metric Manifolds. Mathematics. 7 (2019), 168; doi:10.3390/math7020168.

10. J. A. Oubin̄A: New classes of almost contact metric structures. Publ. Math. Debrecen, 32 (1985), 187-193.

11. A. Sharfuddin and S. I. Hussain: Almost contact structures induced by a conformal transformation. Pub. Inst. Math. 46 (1982), 155-159.

12. S. TANnO: The topology of contact Riemannian manifolds. Illinois J. Math. 12 (1968), 700-717.

13. K. Yano and M. Kon: Structures on Manifolds. Series in Pure Math. Vol 3, World Sci. 1984. 